\title{
Impact Strength of Ferrocement Panel under Low Velocity Impact Loading
}

\author{
Darshan G. Gaidhankar, Mohammad Omid Naqshbandi, Mrudula S. Kulkarni
}

\begin{abstract}
The capability to absorb energy, often called as toughness, is of importance in actual service conditions of mesh reinforced composites, when they may be subjected to static, dynamic and fatigue loads. Toughness evaluated under impact loads is the impact strength. The toughness of materials are determined by two methods, (i) by measuring deformation under impact load, (ii) by determining energy adsorption capacity of materials under impact load. Several methods were used to investigate to determining toughness of materials. In this research work, drop weight impact test were used. The present experimental work describes testing of flat ferrocement panels with different number of layer steel mesh as well as enhancement of panels with steel fiber. The main purpose of this study is to investigate the effect of using different number of wire mesh layer on the flexural strength and impact strength and also effect of varying thickness of panels on the energy absorption of ferrocement panels. The experimental work includes preparation of ferrocement panels reinforced with welded square mesh, woven square mesh with and without hooked steel fibers The ferrocement panels of different sizes were prepared and tested for flexural strength under the two point loading as well as drop weight for impact testing. It is expected that as the mesh layers will be increased the energy absorption capacity of the panel should be increased and the also its effect should be seen for addition of hooked steel fibers.

Keywords: Wire Mesh, Effect, Flexural Strength, Layer, Panel, Ferrocement, Impact Energy
\end{abstract}

\section{INTRODUCTION}

In civil engineering, a high number of civil infrastructures is subjected to serious deterioration as result of chloride attack, carbonation, pollution etc. and many of the civil structures are not considered safe as because of overloading, to maintain the serviceability of older buildings as well to be strengthened and rehabilitation of historical monuments to maintain our cultural heritage. The properties of concrete have shown low tensile strength and low strain capacity, limited ductility, its brittle and the resistance to crack propagation is comparatively low. As per the behavior of RCC structure is ductile, which leaves the structure exposed to ductile failure. Due to ductile failure of concrete, large

Manuscript received on May 22, 2021.

Revised Manuscript received on June 15, 2021.

Manuscript published on June 30, 2021.

* Correspondence Author

Darshan G. Gaidhankar*, Associate Professor, School of Civil Engineering, MIT-World Peace University, Pune (Maharashtra), India. E-mail: darshan.gaidhankar@mitwpu.edu.in

Mohammad Omid Naqshbandi, M. Tech. Civil-Structures Student, MIT World Peace University, Pune (Maharashtra), India. E-mail: Omideng057@gmail.com

M. S. Kulkarni, Professor, SOCE, MIT World Peace University, Pune (Maharashtra), India. Email : mrudulaa.kulkarni@mitwpu.edu.in

(c) The Authors. Published by Blue Eyes Intelligence Engineering and Sciences Publication (BEIESP). This is an open access article under the CC BY-NC-ND license (http://creativecommons.org/licenses/by-nc-nd/4.0/) cracking along with crack widths and deflections that will affect the structures appearance. Therefore, serviceability criteria will be more important to strength. As a result of the problems stated above it is vital to find a new material with improved behavior compared to concrete. In ferrocement, closely spaced multiple layers of mesh or small diameter steel fiber completely infiltrated with, or incapsulated in mortar. In order to improve the behavior of ferrocement, multiple ingredients such as silica fumes, admixtures, fly ash and fibers are also added to it, in general the thickness of ferrocement ranges from $20-100 \mathrm{~mm}$. the fibers reinforced concrete is a composite material, essentially consisting of concrete reinforced by random placement of short discontinuous steel fiber.

\section{Material Constituents of Ferrocement:}

Properties of material for ferrocement including the hydraulic cement mortar, according to the mix design for mortar concrete which include water, sand, wire mesh, Portland cement and admixtures. Water: water is an major ingredient of concrete which should be potable and fit for use as mixing water as well as for curing ferrocement. The quantity and quality of water are required to be looked into very carefully. Sand: fine aggregate give body to the concrete one of the most important factors for producing workable concrete is a good gradation of aggregates. The aggregate should be normal weight, clean, hard, strong free of organic impurities and relatively free of silt and clay. Wire mesh: steel mesh, square welded mesh or chicken wire mesh is made by welding of wire perpendicular with each other which have hexagonal shape as well as some of the mesh filament are galvanized.

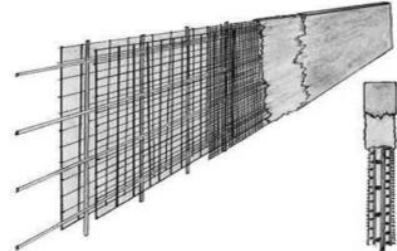

Figure 1. Typical cross section of ferrocement structure

\section{LITERATURE SURVEY}

Subromani R. siva. This study main focus on the behavior of ferro cement reinforcement with waste plastic fiber panels under impact loading. For impact test the result showed that the addition of waste plastic fiber and PCV coated wire mesh layers increased ultimate failure of panels against cracking a total of 8 ferro cement panels with dimension of $600 \mathrm{mmx} 600 \mathrm{~mm} \times 25 \mathrm{~mm}$ and $600 \mathrm{mmx} 400 \mathrm{mmx} 15 \mathrm{~mm}$ were constructed and tested 8 panels tested under low velocity impact.

\section{Published By:}


As reported by the ACI committee 544 is the rig which is used in this research the low velocity impact test was conducted using 1400gm steel ball dropping freely from height $2.4 \mathrm{~m}$ and $1.2 \mathrm{~m}$ thirty two 56 - day age $600 \mathrm{x} 60025 \mathrm{~mm}$ slab and 600x400x15mm slab specimens were tested under low velocity impact. In testing of flexure panels. A special flexural loading frame was exclusively fabricated for testing in order to test the slab on a four point loading at $1 / 3^{\text {rd }}$ span over on effective span of $600 \mathrm{~mm}$ a total of 8 specimens were tested in this investigation on four point loading test Alternatively three category specimens by using of different 1,2 and 3 layers of mesh were tested in this research the proper and the result showed that by increasing of number layers mesh and steel fiber the ultimate strength increase and the crack started from center of top face and propagated on length and width of specimens.

Comingstarful marthong ${ }^{1}$ and deba kumar sarma ${ }^{2}$.The main objective of the experimental work was to study the effect of combing reinforcement steel meshes with discontinuous fibers as reinforcement in this mortar matrix. The experiment done to investigation the flexural behavior of fiber reinforcement ferrocement panels increment 60 plates of size $400 \times 100 \times 12.5 \mathrm{~mm}$ were tested in bending included number of mesh layers and this continues fiber incorporate steel or PET two types of fibers steel and PET specimens were subjected under the different loading. result showed the some number of mesh layers are strongest assembly in both elastic and inelastic ranges shows a good in both flexural strength and energy absorption to failure too spalling of the mortar curve at ultimate load can be very effective with the addition of dis continuous fibers.

Gunasekaran muraliu ${ }^{1}$ Mugahed Amran ${ }^{2}$ Roman Fediuk ${ }^{3}$. The main purpose of this research proper to recognize flexural and low velocity impact response of simply support ferro cement panels reinforced with expanded wire mush and 122 different ferrocement panels was tested against three point flexural load and falling mass impact test. In this experimental work different percentage of 1, 2 and 3\% layers used to comprises with mortar reinforcement. with0.4 water cement ratio was impound for mortar preparation and all panels wire cured in water for 28 days. the investigation was accomplished on the flexural and impact response of ferro cement panels and for the highest compressive strength at 28days was $70.8 \%$ and 50.5 for the incorporating 2 and $1 \%$ dos age of fiber respectively compared to non fiber motors by increasing number of EWM and steel fiber significant flexural strength and ductility index of the ferrocement panels.

AM Shande ${ }^{1}$ and AM Pande ${ }^{2}$ Gulfam Pathan ${ }^{3}$ - The critical investigation accomplished to study the compressive strength of flexural strength slit task strength for m-40 grade of concrete having mix preparation $1: 1$ and $1: 43$ with water cement ratio 0.35 and containing fiber of $0 \% 1 \% 2 \%$ and $3 \%$ volume fraction of hook steel fiber of 50, 60 and 67 aspect ratio were used the cube specimens for compressive strength test $150 \times 150 \mathrm{~mm}$ were cost for $\mathrm{m} 40$ grade of concrete the subs specimens dimension 100x100x500mm were cost for flexural strength of beam and split tensile strength test cylinder specimens of dimension $150 \mathrm{~m}$ and $300 \mathrm{~mm}$ compressive strength, split strength and flexural strength are on higher side for $3 \%$ fiber as compared to that product from $0 \% 1 \%$ and $2 \%$

fibers and also its perceive that compressive strength increase from 11 to $24 \%$ with addition of steel fiber and also its perceive that can compressive strength increase from 11 to $24 \%$ flexural strength increase from 12 to $49 \%$ and split tensile strength increase from 13 to $41 \%$ with addition of steel fiber.

P.B. Sakthivel ${ }^{1}$, A. Ravichandran ${ }^{2}$.This experimental study emphasized and attempt to explore the possibility of adding polyolefin fibers in steel mesh reinforcement cementations composite and conduct low velocity impact test for investigation test specimens of slab size 250 x 250 x $25 \mathrm{~mm}$ were constructed with steel mesh 3 to 5 layers and polyolefin fiber and compared with constructed specimens by having of 3 to 5 layers of steel mesh, statically test were engaged to find out the paired difference in impact energy absorption as well as there were significant different in the energy absorption capacity of cementitious slabs. if steel mesh layers were keeping varied fiber percentage constant the ultimate energy absorption has increased at test to three times when the layers were increase from 3 to 5 layers as well as when $5 \mathrm{~m}$ all amount of polyolefin fiber were tested in small quantities.

\section{OBJECTIVE OF EXPERIMENTAL WORK}

The main purpose of this experimental investigation to study the behavior of ferrocement panels under flexure and impact loading as well as effect of using different layers of steel welded, woven mesh and varying of steel fiber $50 \mathrm{~mm}$ length $1.00 \mathrm{~mm}$ diameter. The several parameters under the consideration of this investigation.

1. Effect of varying steel fiber on flexural strength as well as impact loading.

2. Volume fraction effect on flexural strength of panels.

3. Effect of multiple layers of steel mesh on the energy absorption of slab panels.

\section{EXPERIMENTAL WORK}

In the experimental study flat ferrocement panels constructed and tested under two point loading. First a fall various number of layer steel mesh were tested and then steel fiber used. For Energy absorption capacity drop weight used to collect number of blow for initial and final failure.

\section{A.Materials}

Portland cement grad (43), sand: passing through $2.36 \mathrm{~mm}$ I.S sieve, water, galvanized welded square mesh and galvanized woven mesh diameter of $1.4 \mathrm{~mm}(15 \times 15 \mathrm{~mm}$ opening), steel fiber by having of $50 \mathrm{~mm}$ length $1.00 \mathrm{~mm}$ diameter

\section{B.Mix Proportion}

Cement: sand ratio (1:1.5) water cement ratio $(0.40$ and $0.38)$ Total of 15 panels of size $(70.7 \times 70.7 \times 70.7)$ with and without steel fiber constructed and tested.

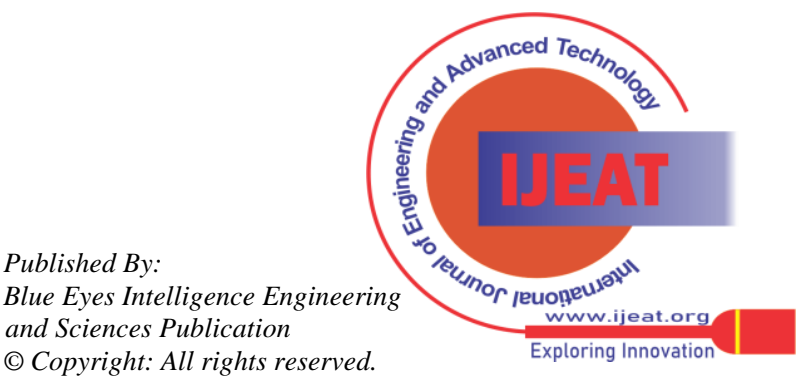


Table 1. Comp. Strength of cubes using Hooked steel fibers

\begin{tabular}{|c|c|c|c|c|}
\hline Sr. No. & $\begin{array}{c}\text { No. of } \\
\text { days }\end{array}$ & $\begin{array}{c}\text { Load at } \\
\text { Failure (KN) }\end{array}$ & $\begin{array}{c}\text { Comp } \\
\text { Strength } \\
\left(\mathrm{N} / \mathrm{mm}^{2}\right)\end{array}$ & $\begin{array}{c}\text { Aver } \\
\text { Comp } \\
\text { Strength } \\
\left(\mathrm{N} / \mathrm{mm}^{2}\right. \\
)\end{array}$ \\
\hline 1 & 28 & 176 & 35.918 & \multirow{2}{*}{36.394} \\
\hline 2 & 28 & 179 & 36.53 & \\
\hline 3 & 28 & 180 & 36.734 & \\
\hline
\end{tabular}

Table 2.Comp. Strength of Cement Mortar Cubes

\begin{tabular}{|c|c|c|c|c|}
\hline $\begin{array}{l}\text { Sr. } \\
\text { No. }\end{array}$ & $\begin{array}{l}\text { No. of } \\
\text { days }\end{array}$ & $\begin{array}{c}\text { Load at } \\
\text { Failure } \\
(\mathrm{KN})\end{array}$ & $\begin{array}{l}\text { Comp. } \\
\text { Strength } \\
\left(\mathrm{N} / \mathrm{mm}^{2}\right)\end{array}$ & $\begin{array}{c}\text { Avg } \\
\text { Comp. } \\
\text { Strength } \\
\left(\mathrm{N} / \mathrm{mm}^{2}\right)\end{array}$ \\
\hline 1. & 07 & 123 & 25.100 & \multirow{3}{*}{25.441} \\
\hline 2. & 07 & 125 & 25.510 & \\
\hline 3. & 07 & 126 & 25.714 & \\
\hline 4. & 28 & 172 & 35.102 & \multirow[b]{3}{*}{35.850} \\
\hline 5. & 28 & 178 & 36.326 & \\
\hline 6. & 28 & 177 & 36.122 & \\
\hline
\end{tabular}

Keyword: Compressive (Comp), Average (Avg)

\section{Casting and Testing Procedure}

The framework properly oiled before costing. Proper content for costing is cement, sand, water and admixture as per the mix design. At first the dry cement and sand were mixed then we putted water and mixed well. Alternatively, we applied one layer of mortar and steel welded mesh or woven mesh by keeping of specified distance between layer of mesh and clear cover. And also $1.5 \%$ in the total weight of specimen steel fiber used in costing for combination of steel fiber and steel welded mesh. . Steel fiber were placed randomly in all the specimen. After 24 hours of costing panels were remove from framework. The all specimen were about to curing for 28 days panel were placed under the testing machine which had $500 \mathrm{~mm}$ space from both ending.

\section{Details of flexural strength}

The flexural strength test under two point loading was constructed on all the panels. During the testing loads and the corresponding deflection are noted down.

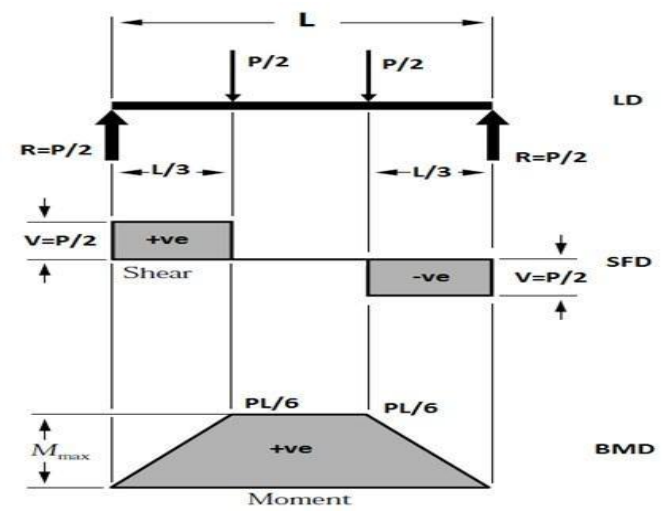

Figure 2. SFD and BMD Under Two Point Loading

Flexural strength was calculated by using the following formula

$$
\frac{\mathrm{M}}{\mathrm{I}}=\frac{\sigma}{\mathrm{Y}} \quad \sigma=\frac{\mathrm{M}}{I} x y
$$

Where, $\mathrm{M}=$ Maximum Bending Moment $=\mathrm{PL} / 6, \mathrm{I}=$ Moment of Inertia $=\mathrm{BD}^{3} / 12, \mathrm{~L}=$ Span

$\mathrm{B}=$ Width of the section, $\mathrm{D}=$ Depth of the sect
Following table are analytical analysis for flexural strength test.

Table 3. Flexural strength of Ferrocement

Panel Size: 500x200x20mm (Experimental)

\begin{tabular}{|c|l|c|c|c|}
\hline \multirow{2}{*}{$\begin{array}{c}\text { Sr. } \\
\text { No. }\end{array}$} & \multirow{2}{*}{ Description } & \multicolumn{3}{|c|}{$\begin{array}{c}\text { Flexural strength a Failure } \\
\text { (MPa) }\end{array}$} \\
\cline { 3 - 5 } & $\begin{array}{c}\text { Layers } \\
02\end{array}$ & $\begin{array}{c}\text { Layers } \\
03\end{array}$ & $\begin{array}{c}\text { Layers } \\
04\end{array}$ \\
\hline 1. & WLD Mesh & 17.5 & 18.5 & 22.5 \\
\hline 2. & $\begin{array}{l}\text { WLD Mesh } \\
\text { with HF }\end{array}$ & 19 & 20 & 25 \\
\hline 3. & WVN Mesh & 15.5 & 15.5 & 16.5 \\
\hline 4. & $\begin{array}{l}\text { WVN Mesh } \\
\text { with HF }\end{array}$ & 16 & 16.9 & 17.5 \\
\hline
\end{tabular}

Table 4. Flexural strength of Ferrocement Panel Size: 700x200x30mm (Experimental)

\begin{tabular}{|c|l|c|c|c|}
\hline \multirow{2}{*}{$\begin{array}{c}\text { Sr. } \\
\text { No. }\end{array}$} & \multirow{2}{*}{ Description } & \multicolumn{3}{|c|}{ Flexural strength a Failure } \\
\cline { 3 - 5 } & & $\begin{array}{c}02 \\
\text { Layers }\end{array}$ & $\begin{array}{c}03 \\
\text { Layers }\end{array}$ & $\begin{array}{c}04 \\
\text { Layers }\end{array}$ \\
\hline 1. & WLD Mesh & 15 & 16 & 17.3 \\
\hline 2. & $\begin{array}{l}\text { WLD Mesh } \\
\text { with HF }\end{array}$ & 15.6 & 16.66 & 19.6 \\
\hline 3. & WVN Mesh & 13 & 13.6 & 16.66 \\
\hline 4. & $\begin{array}{l}\text { WVN Mesh } \\
\text { with HF }\end{array}$ & 14 & 14.5 & 15.6 \\
\hline
\end{tabular}

Table 5. Flexural strength of Ferrocement Panels Panels Size: 800x200x40mm(Experimental)

\begin{tabular}{|c|l|c|c|c|}
\hline \multirow{2}{*}{$\begin{array}{c}\text { Sr. } \\
\text { No. }\end{array}$} & \multirow{2}{*}{ Description } & \multicolumn{3}{|c|}{ Flexural strength at Failure } \\
& & $\begin{array}{c}\text { Layers } \\
02\end{array}$ & $\begin{array}{c}\text { Layers } \\
03\end{array}$ & $\begin{array}{c}\text { Layers } \\
04\end{array}$ \\
\cline { 3 - 5 } & & 11.7 & 11.9 & 13.3 \\
\hline 1. & WLD Mesh & 12.03 & 12.4 & 14.7 \\
\hline 2. & $\begin{array}{l}\text { WLD Mesh } \\
\text { with HF }\end{array}$ & 9 & 10 & 10.3 \\
\hline 3. & WVN Mesh & 10.06 & 10.4 & 11.2 \\
\hline 4. & $\begin{array}{l}\text { WVN Mesh } \\
\text { with HF }\end{array}$ & & & \\
\hline
\end{tabular}

\section{TEST RESULT}

Energy Absorption of Ferrocement Panels:

Calculation for finding energy absorption capacity of ferrocement panel at initial, final crack.

$E_{\text {imp }}=N \cdot g \cdot h \cdot m$

$E_{\text {imp }}=$ energy impact

$N=$ Number of blow

$h=$ Hieght of drop

$m=$ Mass of arop weight

Following table are for Energy Absorption of ferrocement panels test.

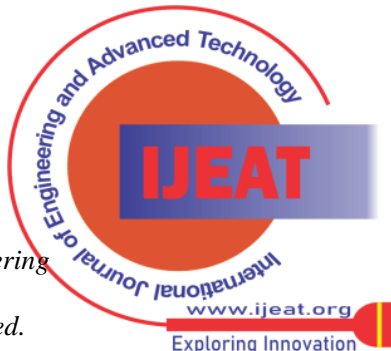


Table 6. Number of blows For Ferrocement Panels 250 x 250 (For $h=0.5 m)$

\begin{tabular}{|c|l|c|c|c|}
\hline \multirow{2}{*}{$\begin{array}{c}\text { Sr. } \\
\text { No. }\end{array}$} & \multirow{3}{*}{ Description } & \multicolumn{3}{|c|}{ Number of Blow For Initial Failure } \\
\cline { 3 - 5 } & & $\begin{array}{c}\text { Layer } \\
02\end{array}$ & $\begin{array}{c}\text { Layer } \\
03\end{array}$ & $\begin{array}{c}\text { Layer } \\
04\end{array}$ \\
\hline 1. & $20 \mathrm{~mm}$ WLD & 15 & 16 & 22 \\
\hline 2. & $30 \mathrm{~mm}$ WLD & 17 & 19 & 24 \\
\hline 3. & $40 \mathrm{~mm}$ WLD & 21 & 23 & 27 \\
\hline 4. & $\begin{array}{l}\text { 20mm WLD } \\
\text { with HF }\end{array}$ & 16 & 18 & 22 \\
\hline 5. & $\begin{array}{l}30 \mathrm{~mm} \text { WLD } \\
\text { with HF }\end{array}$ & 17 & 20 & 25 \\
\hline 6. & $\begin{array}{l}40 \mathrm{~mm} \text { WLD } \\
\text { with HF }\end{array}$ & 22 & 23 & 29 \\
\hline
\end{tabular}

Table 7. Number of blow for ferrocement Panels $250 \times 250$ (For $h=0.5 \mathrm{~m})$

\begin{tabular}{|c|l|c|c|c|}
\hline \multirow{2}{*}{$\begin{array}{c}\text { Sr. } \\
\text { No. }\end{array}$} & \multirow{2}{*}{ Description } & \multicolumn{3}{|c|}{ Number of Blow For final } \\
\cline { 3 - 5 } & & $\begin{array}{c}\text { Layer } \\
02\end{array}$ & $\begin{array}{c}\text { Layer } \\
03\end{array}$ & $\begin{array}{c}\text { Layer } \\
04\end{array}$ \\
\hline 1 & $20 \mathrm{~mm}$ WLD & 98 & 112 & 122 \\
\hline 2 & $30 \mathrm{~mm}$ WLD & 128 & 134 & 147 \\
\hline 3 & $40 \mathrm{~mm}$ WLD & 168 & 182 & 210 \\
\hline 4 & $\begin{array}{l}20 \mathrm{~mm} \text { WLD with } \\
\text { HF }\end{array}$ & 104 & 118 & 126 \\
\hline 5 & $\begin{array}{l}30 \mathrm{~mm} \text { WLD with } \\
\text { HF }\end{array}$ & 138 & 136 & 155 \\
\hline 6 & $\begin{array}{l}40 \mathrm{~mm} \text { WLD with } \\
\text { HF }\end{array}$ & 175 & 190 & 221 \\
\hline
\end{tabular}

Table 8.Initial energy absorption table For Ferrocement Panels Size 250 x 250 (For $h=0.5 \mathrm{~m})$

\begin{tabular}{|c|l|c|c|c|}
\hline \multirow{2}{*}{$\begin{array}{c}\text { Sr. } \\
\text { No. }\end{array}$} & \multirow{2}{*}{ Description } & \multicolumn{3}{|c|}{$\begin{array}{c}\text { Initial Energy Absorption of } \\
\text { Ferrocement Panels }\end{array}$} \\
\cline { 3 - 5 } & $\begin{array}{c}\text { Layer } \\
02\end{array}$ & $\begin{array}{c}\text { Layer } \\
02\end{array}$ & $\begin{array}{c}\text { Layer } \\
02\end{array}$ \\
\hline 1. & $20 \mathrm{~mm}$ WLD & 257.51 & 274.68 & 394.9 \\
\hline 2. & $30 \mathrm{~mm}$ WLD & 291.85 & 326.18 & 412.02 \\
\hline 3. & $40 \mathrm{~mm}$ WLD & 306.6 & 394.86 & 463.52 \\
\hline 4. & $\begin{array}{l}\text { 20mm WL } \\
\text { with HF }\end{array}$ & 274.68 & 309.02 & 373.69 \\
\hline 5. & $\begin{array}{l}30 \mathrm{~mm} \text { WLD } \\
\text { with HF }\end{array}$ & 291.85 & 343.4 & 429.19 \\
\hline 6. & $\begin{array}{l}40 \mathrm{~mm} \text { WLD } \\
\text { with HF }\end{array}$ & 377.69 & 394.9 & 497.86 \\
\hline
\end{tabular}

Table 9. Final Energy Absorption of Ferrocement Panels 250 x 250 (For $h=0.5 \mathrm{~m})$

\begin{tabular}{|c|c|c|c|c|}
\hline \multirow[t]{2}{*}{$\begin{array}{l}\text { Sr. } \\
\text { No. }\end{array}$} & \multirow[t]{2}{*}{ Description } & \multicolumn{3}{|c|}{$\begin{array}{c}\text { Final Energy Absorption of } \\
\text { Ferrocement Panels }\end{array}$} \\
\hline & & $\begin{array}{c}\text { Layer } \\
02\end{array}$ & $\begin{array}{c}\text { Layer } \\
03\end{array}$ & Layer 04 \\
\hline 1 & 20mm WLD & 1682.42 & 1922.76 & 2094.4 \\
\hline 2 & 30mm WLD & 2179.44 & 2300.46 & 2523.62 \\
\hline 3 & 40mm WLD & 2884.14 & 3124.49 & 3605.18 \\
\hline 4 & $\begin{array}{l}\text { 20mm WL } \\
\text { with HF }\end{array}$ & 1785.42 & 2025.77 & 2163.11 \\
\hline 5 & $\begin{array}{l}\text { 30mm WLD } \\
\text { with HF }\end{array}$ & 2369.12 & 2334.78 & 2660.96 \\
\hline 6 & $\begin{array}{l}\text { 40mm WLD } \\
\text { with HF }\end{array}$ & 3004.31 & 3261.83 & 3794.01 \\
\hline
\end{tabular}

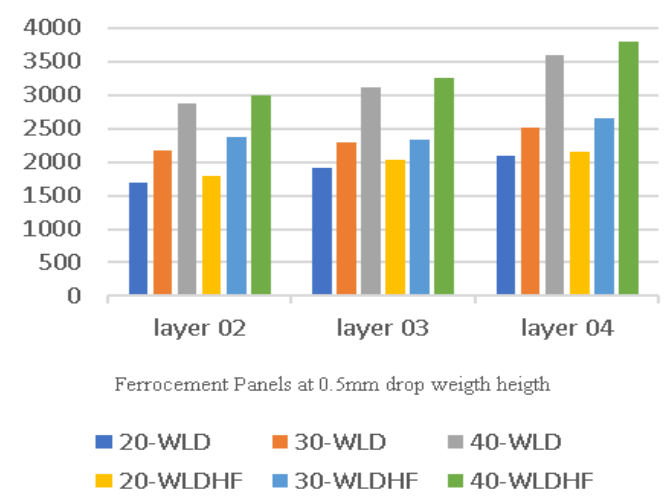

Graph 1. For final Energy absorption of ferrocement panels

Table 10. Number of blows For Ferrocement Panels 250 x 250 (For h = 1m)

\begin{tabular}{|c|l|c|c|c|}
\hline \multirow{2}{*}{$\begin{array}{c}\text { Sr. } \\
\text { No. }\end{array}$} & \multirow{2}{*}{ Description } & \multicolumn{3}{|c|}{ Number of Blow For Initial } \\
\cline { 3 - 5 } & $\begin{array}{c}\text { Layer } \\
02\end{array}$ & $\begin{array}{c}\text { Layer } \\
02\end{array}$ & $\begin{array}{c}\text { Layer } \\
02\end{array}$ \\
\hline 1. & $20 \mathrm{~mm}$ WLD & 9 & 9 & 11 \\
\hline 2. & $30 \mathrm{~mm}$ WLD & 9 & 10 & 11 \\
\hline 3. & $40 \mathrm{~mm}$ WLD & 10 & 10 & 12 \\
\hline 4. & $\begin{array}{l}\text { 20mm WL } \\
\text { with HF }\end{array}$ & 9 & 9 & 12 \\
\hline 5. & $\begin{array}{l}30 \mathrm{~mm} \text { WLD } \\
\text { with HF }\end{array}$ & 10 & 10 & 13 \\
\hline 6. & $\begin{array}{l}40 \mathrm{~mm} \text { WLD } \\
\text { with HF }\end{array}$ & 11 & 10 & 13 \\
\hline
\end{tabular}

Table 11.Number of blow for ferrocement Panels 250 x 250 (For $h=1 \mathrm{~m})$

\begin{tabular}{|c|l|c|c|c|}
\hline \multirow{2}{*}{$\begin{array}{c}\text { Sr. } \\
\text { No. }\end{array}$} & \multirow{2}{*}{ Description } & \multicolumn{3}{|c|}{ Number of Blow For final } \\
\cline { 3 - 5 } & $\begin{array}{c}\text { Layer } \\
02\end{array}$ & $\begin{array}{c}\text { Layer } \\
03\end{array}$ & $\begin{array}{c}\text { Layer } \\
04\end{array}$ \\
\hline 1 & 20mm WLD & 45 & 50 & 53 \\
\hline 2 & $30 \mathrm{~mm}$ WLD & 52 & 57 & 64 \\
\hline 3 & $40 \mathrm{~mm}$ WLD & 66 & 72 & 83 \\
\hline 4 & $\begin{array}{l}\text { 20mm WL } \\
\text { with HF }\end{array}$ & 56 & 63 & 72 \\
\hline 5 & $\begin{array}{l}30 \mathrm{~mm} \text { WLD } \\
\text { with HF }\end{array}$ & 60 & 65 & 86 \\
\hline 6 & $\begin{array}{l}40 \mathrm{~mm} \text { WLD } \\
\text { with HF }\end{array}$ & 72 & 85 & 95 \\
\hline
\end{tabular}

Table 12. Initial energy absorption table For Ferrocement

Panels 250 x 250 (For $h=1 \mathrm{~m})$

\begin{tabular}{|c|l|c|c|c|}
\hline \multirow{2}{*}{$\begin{array}{c}\text { Sr. } \\
\text { No. }\end{array}$} & \multirow{2}{*}{ Description } & \multicolumn{3}{|c|}{$\begin{array}{c}\text { Initial Energy Absorption of } \\
\text { Ferrocement Panels }\end{array}$} \\
\cline { 3 - 5 } & $\begin{array}{c}\text { Layer } \\
02\end{array}$ & $\begin{array}{c}\text { Layer } \\
03\end{array}$ & Layer 04 \\
\hline 1 & $20 \mathrm{~mm}$ WLD & 309.02 & 309.02 & 373.69 \\
\hline 2 & $30 \mathrm{~mm}$ WLD & 309.02 & 343.4 & 373.69 \\
\hline 3 & $40 \mathrm{~mm}$ WLD & 343.04 & 343.4 & 412.02 \\
\hline 4 & $\begin{array}{l}20 \mathrm{~mm} \text { WL } \\
\text { with HF }\end{array}$ & 309.02 & 309.02 & 412 \\
\hline
\end{tabular}

Published By: Blue Eyes Intelligence Engineering and Sciences Publication (C) Copyright: All rights reserved. 


\begin{tabular}{|c|l|c|c|c|}
\hline 5 & $\begin{array}{l}\text { 30mm WLD } \\
\text { with HF }\end{array}$ & 343.04 & 343.4 & 446.36 \\
\hline 6 & $\begin{array}{l}\text { 40mm WLD } \\
\text { with HF }\end{array}$ & 373.69 & 343.4 & 446.36 \\
\hline
\end{tabular}

Table 13.Final energy absorption table For Ferrocement Panels 250 x 250 (For $h=1 \mathrm{~m})$

\begin{tabular}{|c|c|c|c|c|}
\hline \multirow[t]{2}{*}{$\begin{array}{l}\text { Sr. } \\
\text { No. }\end{array}$} & \multirow[t]{2}{*}{ Description } & \multicolumn{3}{|c|}{$\begin{array}{l}\text { Final Energy Absorption of } \\
\text { Ferrocement Panels }\end{array}$} \\
\hline & & $\begin{array}{c}\text { Layer } \\
02\end{array}$ & $\begin{array}{l}\text { Layer } \\
03\end{array}$ & Layer 04 \\
\hline 1 & $20 \mathrm{~mm}$ WLD & 1545.1 & 1716.8 & 2163.12 \\
\hline 2 & 30mm WLD & 1785.42 & 1957.1 & 2197.44 \\
\hline 3 & 40mm WLD & 2265.11 & 2472.12 & 2849.8 \\
\hline 4 & $\begin{array}{l}\text { 20mm WL } \\
\text { with HF }\end{array}$ & 1922.76 & 2163.12 & 2472.12 \\
\hline 5 & $\begin{array}{l}30 \mathrm{~mm} \text { WLD } \\
\text { with HF }\end{array}$ & 2060.1 & 2231.76 & 2952.81 \\
\hline 6 & $\begin{array}{l}40 \mathrm{~mm} \text { WLD } \\
\text { with HF }\end{array}$ & 2472.12 & 2918.48 & 3261.86 \\
\hline
\end{tabular}

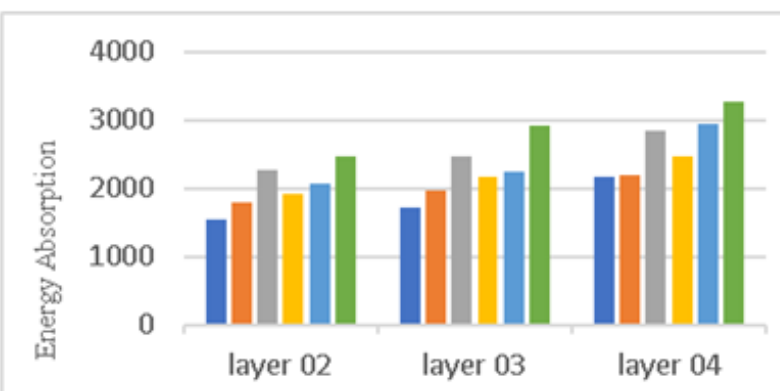

Ferrocement $P$ anels at $1 \mathrm{~mm}$ drop weigth heigth

a 20-WLD $=30$-WLD $=40$-WLD

$\approx$ 20-WLDHF $\approx 30-$ WLDHF $\quad$ 40-WLDHF

Graph 2. For final Energy absorption of ferrocement panels

Table 14. Number of blows For Ferrocement Panels250 x 250 (For h $=0.5 \mathrm{~m})$

\begin{tabular}{|c|c|c|c|c|}
\hline \multirow{2}{*}{$\begin{array}{c}\text { Sr. } \\
\text { No. }\end{array}$} & \multirow{2}{*}{ Description } & \multicolumn{3}{|c|}{ Number of Blow For Initial } \\
\cline { 3 - 5 } & $\begin{array}{c}\text { Layer } \\
02\end{array}$ & $\begin{array}{c}\text { Layer } \\
03\end{array}$ & $\begin{array}{c}\text { Layer } \\
04\end{array}$ \\
\hline 1 & $20 \mathrm{~mm}$ WVN & 12 & 13 & 17 \\
\hline 2 & $30 \mathrm{~mm}$ WVN & 12 & 13 & 17 \\
\hline 3 & $40 \mathrm{~mm}$ WVN & 13 & 14 & 18 \\
\hline 4 & $\begin{array}{c}20 \mathrm{~mm} \text { WVN } \\
\text { with HF }\end{array}$ & 13 & 14 & 18 \\
\hline 5 & $\begin{array}{c}30 \mathrm{~mm} \text { WVN } \\
\text { with HF }\end{array}$ & 14 & 14 & 19 \\
\hline 6 & $\begin{array}{c}40 \mathrm{~mm} \text { WVN } \\
\text { with HF }\end{array}$ & 14 & 15 & 19 \\
\hline
\end{tabular}

Table 15. Number of blow for ferrocement Panels 250 x 250 (For $h=0.5 \mathrm{~m}$ )

\begin{tabular}{|c|c|c|c|c|}
\hline \multirow{2}{*}{$\begin{array}{c}\text { Sr. } \\
\text { No. }\end{array}$} & \multirow{2}{*}{ Description } & \multicolumn{3}{|c|}{ Number of Blow For final } \\
\cline { 3 - 5 } & & $\begin{array}{c}\text { Layer } \\
02\end{array}$ & $\begin{array}{c}\text { Layer } \\
03\end{array}$ & Layer 04 \\
\cline { 3 - 5 } & & 88 & 96 & 106 \\
\hline 1 & $20 \mathrm{~mm}$ WVN & 112 & 121 & 128 \\
\hline 2 & $30 \mathrm{~mm}$ WVN & \multicolumn{3}{|c}{} \\
\hline
\end{tabular}

\begin{tabular}{|c|l|c|c|c|}
\hline 3 & $40 \mathrm{~mm}$ WVN & 146 & 160 & 175 \\
\hline 4 & $\begin{array}{l}20 \mathrm{~mm} \text { WVN } \\
\text { with HF }\end{array}$ & 92 & 99 & 110 \\
\hline 5 & $\begin{array}{l}30 \mathrm{~mm} \text { WVN } \\
\text { with HF }\end{array}$ & 118 & 127 & 135 \\
\hline 6 & $\begin{array}{l}40 \mathrm{~mm} \text { WVN } \\
\text { with HF }\end{array}$ & 162 & 172 & 189 \\
\hline
\end{tabular}

Table 16.Initial Energy Absorption For Ferrocement Panels250 x 250 (For h $=0.5 \mathrm{~m}$ )

\begin{tabular}{|c|l|c|c|c|}
\hline $\begin{array}{c}\text { Sr. } \\
\text { No. }\end{array}$ & \multirow{2}{*}{ Description } & \multicolumn{2}{|c|}{$\begin{array}{c}\text { Initial Energy Absorption of } \\
\text { Ferrocement Panels }\end{array}$} \\
\cline { 3 - 5 } & & $\begin{array}{c}\text { Layer } \\
02\end{array}$ & $\begin{array}{c}\text { Layer } \\
03\end{array}$ & $\begin{array}{c}\text { Layer } \\
04\end{array}$ \\
\hline 1 & $20 \mathrm{~mm} \mathrm{WVN}$ & 206.01 & 223.18 & $\begin{array}{c}291.8 \\
5\end{array}$ \\
\hline 2 & $30 \mathrm{~mm} \mathrm{WVN}$ & 206.01 & 223.18 & $\begin{array}{c}291.8 \\
5\end{array}$ \\
\hline 3 & $40 \mathrm{~mm} \mathrm{WVN}$ & 223.18 & 240.35 & $\begin{array}{c}309.0 \\
2\end{array}$ \\
\hline 4 & $20 \mathrm{~mm} \mathrm{WVN}$ & 223.18 & 240.35 & $\begin{array}{c}309.0 \\
2\end{array}$ \\
\hline 5 & $\begin{array}{l}30 \mathrm{~mm} \text { WVN } \\
\text { with HF }\end{array}$ & 240.35 & 240.35 & $\begin{array}{c}326.1 \\
8\end{array}$ \\
\hline 6 & $\begin{array}{l}40 \mathrm{~mm} \mathrm{WVN} \\
\text { with HF }\end{array}$ & 240.35 & 257.51 & $\begin{array}{c}326.1 \\
8\end{array}$ \\
\hline
\end{tabular}

Table 17. Final Energy Absorption ferrocement Panels 250 x 250 (For h = 0.5m)

\begin{tabular}{|c|l|c|c|c|}
\hline \multirow{2}{*}{$\begin{array}{c}\text { Sr. } \\
\text { No. }\end{array}$} & \multirow{2}{*}{ Description } & \multicolumn{3}{|c|}{$\begin{array}{c}\text { Final Energy Absorption of } \\
\text { Ferrocement Panels }\end{array}$} \\
\cline { 3 - 5 } & $\begin{array}{c}\text { Layer } \\
02\end{array}$ & $\begin{array}{c}\text { Layer } \\
03\end{array}$ & $\begin{array}{c}\text { Layer } \\
04\end{array}$ \\
\hline 1 & $20 \mathrm{~mm}$ WVN & 1510.74 & 1648.08 & 1819.8 \\
\hline 2 & $30 \mathrm{~mm}$ WVN & 1922.76 & 2077.27 & 3124.4 \\
\hline 3 & $40 \mathrm{~mm}$ WVN & 2506.5 & 2746.8 & 3004.3 \\
\hline 4 & $\begin{array}{l}20 \mathrm{~mm} \text { WVN } \\
\text { with HF }\end{array}$ & 1579.41 & 1699.58 & 1888.4 \\
\hline 5 & $\begin{array}{l}30 \mathrm{~mm} \text { WVN } \\
\text { with HF }\end{array}$ & 2025.77 & 2180.27 & 2317.6 \\
\hline 6 & $\begin{array}{l}40 \mathrm{~mm} \text { WVN } \\
\text { with HF }\end{array}$ & 2781.14 & 2952.81 & 3244 \\
\hline
\end{tabular}

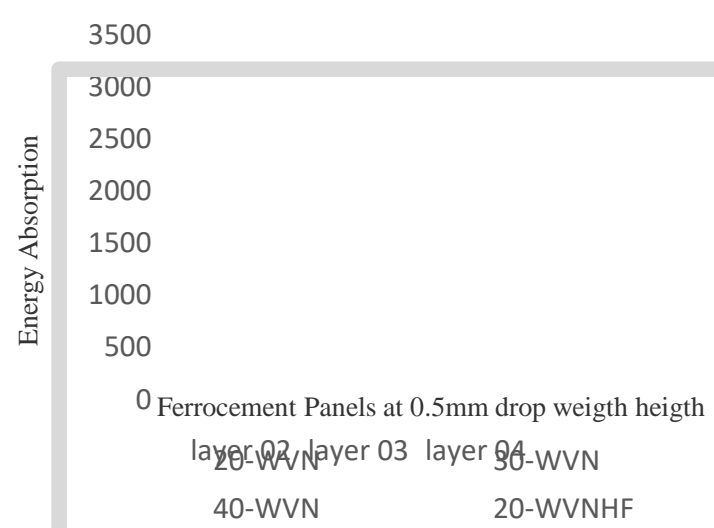

Graph 3. For final Energy absorption of ferrocement panels

Published By:

Blue Eyes Intelligence Engineering and Sciences Publication

(c) Copyright: All rights reserved.

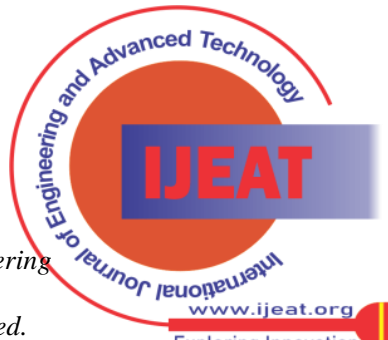


Table 18.Number of blow for ferrocement Panels 250 x 250 (For h = 1m)

\begin{tabular}{|c|l|c|c|c|}
\hline \multirow{2}{*}{$\begin{array}{c}\text { Sr. } \\
\text { No. }\end{array}$} & \multirow{2}{*}{ Description } & \multicolumn{3}{|c|}{ Fuilure } \\
\cline { 3 - 5 } & & $\begin{array}{c}\text { Layer } \\
02\end{array}$ & $\begin{array}{c}\text { Layer } \\
03\end{array}$ & $\begin{array}{c}\text { Layer } \\
04\end{array}$ \\
\hline 1 & $20 \mathrm{~mm} \mathrm{WVN}$ & 07 & 09 & 10 \\
\hline 2 & $30 \mathrm{~mm}$ WVN & 07 & 10 & 11 \\
\hline 3 & $40 \mathrm{~mm}$ WVN & 08 & 10 & 11 \\
\hline 4 & $20 \mathrm{~mm}$ WVN with HF & 08 & 10 & 12 \\
\hline 5 & $30 \mathrm{~mm}$ WVN with HF & 09 & 11 & 13 \\
\hline 6 & $40 \mathrm{~mm}$ WVN with HF & 09 & 11 & 13 \\
\hline
\end{tabular}

Table 19.Number of blow for ferrocement Panels 250 x $250($ For $h=1 \mathrm{~m})$

\begin{tabular}{|c|l|c|c|c|}
\hline \multirow{2}{*}{$\begin{array}{c}\text { Sr. } \\
\text { No. }\end{array}$} & \multirow{2}{*}{ Description } & \multicolumn{3}{|c|}{ Fuilure } \\
\cline { 3 - 5 } & & $\begin{array}{c}\text { Layer } \\
02\end{array}$ & $\begin{array}{c}\text { Layer } \\
03\end{array}$ & $\begin{array}{c}\text { Layer } \\
04\end{array}$ \\
\hline 1 & $20 \mathrm{~mm} \mathrm{WVN}$ & 42 & 45 & 49 \\
\hline 2 & $30 \mathrm{~mm}$ WVN & 49 & 56 & 61 \\
\hline 3 & $40 \mathrm{~mm}$ WVN & 61 & 75 & 84 \\
\hline 4 & $20 \mathrm{~mm}$ WVN with HF & 45 & 53 & 59 \\
\hline 5 & $30 \mathrm{~mm}$ WVN with HF & 57 & 62 & 73 \\
\hline 6 & $40 \mathrm{~mm}$ WVN with HF & 69 & 79 & 95 \\
\hline
\end{tabular}

Table 20. Initial Energy Absorption For Ferrocement Panels 250 x 250 (For $h=1 \mathrm{~m})$

\begin{tabular}{|c|l|c|c|c|}
\hline $\begin{array}{c}\text { Sr. } \\
\text { No. }\end{array}$ & \multirow{2}{*}{ Description } & \multicolumn{3}{|c|}{$\begin{array}{c}\text { Initial Energy Absorption of } \\
\text { Ferrocement Panels }\end{array}$} \\
\cline { 3 - 5 } & $\begin{array}{c}\text { Layer } \\
02\end{array}$ & $\begin{array}{c}\text { Layer } \\
03\end{array}$ & Layer 04 \\
\hline 1 & $20 \mathrm{~mm}$ WVN & 240.35 & 309.02 & 343.35 \\
\hline 2 & $30 \mathrm{~mm} \mathrm{WVN}$ & 240.35 & 343.35 & 377.69 \\
\hline 3 & $40 \mathrm{~mm}$ WVN & 274.68 & 343.35 & 377.69 \\
\hline 4 & $\begin{array}{l}20 \mathrm{~mm} \mathrm{WVN} \\
\text { with HF }\end{array}$ & 274.68 & 343.35 & 412.02 \\
\hline 5 & $\begin{array}{l}30 \mathrm{~mm} \text { WVN } \\
\text { with HF }\end{array}$ & 309.02 & 377.69 & 446.36 \\
\hline 6 & $\begin{array}{l}40 \mathrm{~mm} \text { WVN } \\
\text { with HF }\end{array}$ & 309.02 & 377.69 & 440.36 \\
\hline
\end{tabular}

Table 21 Final Energy Absorption For Ferrocement Panels $250 \times 250$ ( For $h=1 \mathrm{~m}$ )

\begin{tabular}{|c|c|c|c|c|}
\hline \multirow[t]{2}{*}{$\begin{array}{l}\text { Sr. } \\
\text { No. }\end{array}$} & \multirow[t]{2}{*}{ Description } & \multicolumn{3}{|c|}{$\begin{array}{l}\text { Final Energy Absorption of } \\
\text { Ferrocement Panels }\end{array}$} \\
\hline & & $\begin{array}{l}\text { Layer } \\
02\end{array}$ & $\begin{array}{l}\text { Layer } \\
03\end{array}$ & Layer 04 \\
\hline 1 & $20 \mathrm{~mm} \mathrm{WVN}$ & 1442.1 & 1545.08 & 1682.42 \\
\hline 2 & $30 \mathrm{~mm}$ WVN & 1682.42 & 1922.76 & 2094.44 \\
\hline 3 & $40 \mathrm{~mm} \mathrm{WVN}$ & 2094.44 & 2575.13 & 2884.14 \\
\hline 4 & $\begin{array}{l}\text { 20mm WVN } \\
\text { with HF }\end{array}$ & 1545.08 & 1819.8 & 2025.77 \\
\hline 5 & $\begin{array}{l}\text { 30mm WVN } \\
\text { with HF }\end{array}$ & 1957.1 & 2128.8 & 2506.5 \\
\hline 6 & $\begin{array}{l}\text { 40mm WVN } \\
\text { with HF }\end{array}$ & 2369.12 & 2712.47 & 3261.83 \\
\hline
\end{tabular}

\section{Graph 4. For final Energy absorption of ferrocement panels}

Nomenclatures used : FC - Ferrocement Panel WLD - FC panel with welded mesh WVN - FC panel with woven mesh WLD with HF - FC panel welded mesh and Hooked Fibres WVN with HF - FC panel woven mesh and hooked fibres

\section{CONCLUSION}

An excellent investigation was conducted on energy absorption of ferrocement panels in different number of wire mesh with steel fiber and without steel fiber. as well different drop height has been in the cover of the investigation. All finding from experimental and analytical investigation the following main conclusion.

1) By increasing the height of drop of hammer energy absorption capacity of panel decreases.

2) Increasing the thickness of panel increases energy absorption capacity of panel.

3) The energy absorption capacity of ferrocement panel using welded mesh with hooked fibres shows excellent results than using woven mesh with hooked fibres .

4) Addition of fibers in mortar shows slight increases in compressive strength of mortar.

5) Increase in number of wire mesh layers increases the flexural strength of ferrocement panels.

6) Increase in thickness of ferrocement panel shows reduction in the flexural strength.

\section{REFERENCES}

1. Abdulkader Ismail A. Al-Hadithi, Khalil Ibrahim Aziz, Mohammed Tarrad Nawar Al-Dulaimi,"Behaviour of Ferro-cement slabs containing SBR under impact loads", International Journal of

2. Gunasekaran Murali, Mugahed Amran, Roman Fediuk, Nikolai atin,Sudharshan N. Raman , Gundu Maithreyi and Arunachalam Sumathi, Experimental Study On Flexural And Impact Behavior Of Ferrocement Slabs

3. Darshan G. Gaidhankar, M.S. Kulkarni,"Ferrocement Panels Under Impact Loading By Incorporating Basalt Fibres", International Journal of Scientific \& Technology Research (IJSTR), Volume -09, Issue-06, June 2020, Pg. 678-682, ISSN 2277-8616

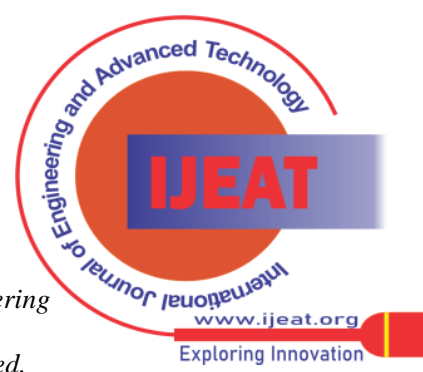


4. T.Subramani, R.Siva. "Experimental Study On Flexural And Impact Behavior Of Ferro-cement Slabs", International Journal of Application or Innovation in Engineering \& Management (IJAIEM) , Volume 5, Issue 5, May 2016

5. Darshan G. Gaidhankar, M.S. Kulkarni, "Ferrocement Panels Under Flexure By Partial Replacement of Cement With Marble Powder", International Journal of Innovative Technology and Exploring Engineering (IJITEE), Volume -09, Issue-8, June 2020, Pg. 255-260, ISSN: 2278-3075

6. "Ferrocement and Laminated Cementitious Composites", a book by Antoine A. Naaman

7. Darshan G. Gaidhankar, M.S. Kulkarni,"Ferrocement Panels Under High Velocity Impact By Incorporating Waste Foundry Sand", International Journal of Engineering and Advanced Technology (IJEAT), Volume -09, Issue-05, June 2020, Pg. 1174-1182, ISSN 2249-8958

8. Ferrocement Construction and Design - A Construction Mannual, Book by Dr. B. N. Divekar

9. Darshan G. Gaidhankar, M.S. Kulkarni, "Behaviour of Ferrocement Panel Under Impact Loading", International Refereed Journal of Engineering and Science, Vol. 07, Issue-07, July 2018, Pg. 52-61, ISSN 2319- 1821.

10. Darshan G. Gaidhankar"Effect of Mortar Strength On The Behaviour of Ferrocement Panel Under Low Velocity Impact ", International Journal of Innovative Technology and Exploring Engineering (IJITEE), Volume -08, Issue-12, October 2019, Pg. 5245-5249, ISSN: 2278-3075

11. "Design of Ferrocement Structures" a book by Dr. B. N. Divekar

12. Darshan G. Gaidhankar, M. S. Kulkarni, "Behaviour of Ferrocement Panels Using Welded Square Mesh ", International Journal For Research and Development In Technology, Vol. 8, Issue-04, October 2017, Pg. 138-150, ISSN 2349- 3585.

13. M. S. Kulkarni, "Comparative performance of Precast Ferrocement panel wall system with Brick wall, under static and Impact load, an application for war-prone housing", IJLTET ,e-ISSN : 2278-621X p-ISSN : 2319-3778/Vol.12,Issue.1,Pp:1-8/2019

14. Darshan G. Gaidhankar, M. S. Kulkarni, "Ferrocement Composite Members Under Uniaxial Compression Using LGS Channel", International Journal of Scientific \& Technology Research (IJSTR), Volume -08, Issue-09, September 2019, Pg. 516-519, ISSN 2277-8616

15. Darshan G. Gaidhankar, M. S. Kulkarni, "Analysis of Curved Ferrocement Panels ", International Journal For Science and Research in Technology, Vol. 3, Issue-10, October 2017, Pg. 253-257, ISSN 2395- 1052

16. Darshan G. Gaidhankar, "Effect of Different Types of Fibers on the Flexural Strength of Ferrocement Panels", International Journal of Innovative Research in Science, Engineering and Technology, Vol. 5 , Issue-9, September 2016, Pg. 16991-16996 (IJIRSET) , ISSN 2319-8753.

17. Darshan G. Gaidhankar,"Comparative Study of Ferro-Cement Pane Using Welded Square Mesh and Expanded Mesh", International Journal of Innovative Science, Engineering \& Technology (IJISET), Vol. 2 Issue 6, Pg. 594-597, June 2015, ISSN 2348 - 7968.

\section{AUTHORS PROFILE}

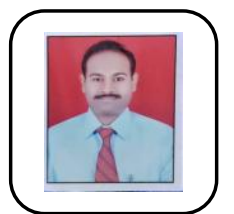

Dr. Darshan G. Gaidhankar, Ph.D. in Civil Engineering, Working as a Associate Professor in MIT-WPU, Pune. Published more than 20 research papers in reputed journals. Research area is Ferrocement Technology, Composite Materials, Concrete Technology and Earthquake Engineering

Email: darshan.gaidhankar@mitwpu.edu.in

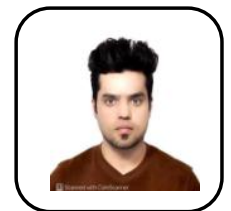

Mohammad Omid Naqshbandi, M. Tech. Structural Engineering Student Very much interesting in learning development of new construction materials and technologies.

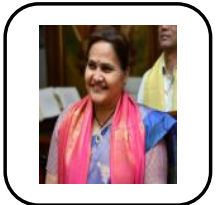

Dr. Mrudula S. Kulkarni, Ph.D. in Civil Engineering, Working as a Professor in MIT-WPU, Pune. Published more than 30 research papers in reputed journals. Research area is Bio-Mechanics, Finite Element Analysis, Ferrocement Technology and Composite

Published By: Blue Eyes Intelligence Engineering and Sciences Publication

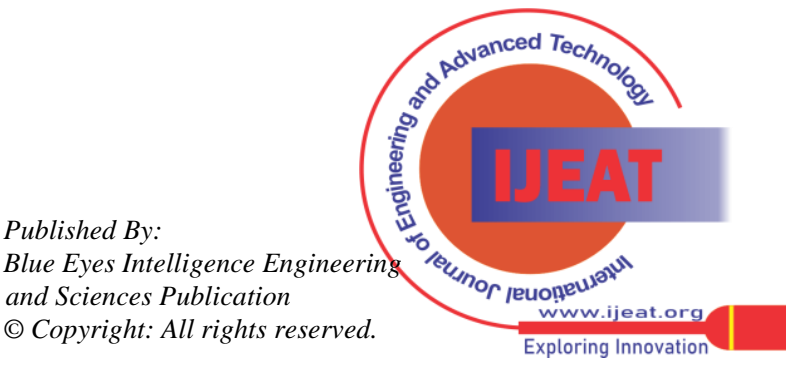

